\title{
Optical tuning of photonic bandgaps in dye-doped nematic liquid crystal photonic crystal fibers
}

Alkeskjold, Thomas Tanggaard; Bjarklev, Anders Overgaard; Hermann, David Sparre; Broeng, Jes; Li, J.; Wu, S-.T.

Published in:

OFC Proceedings

Publication date:

2005

Document Version

Publisher's PDF, also known as Version of record

Link back to DTU Orbit

Citation (APA):

Alkeskjold, T. T., Bjarklev, A. O., Hermann, D. S., Broeng, J., Li, J., \& Wu, S-T. (2005). Optical tuning of photonic bandgaps in dye-doped nematic liquid crystal photonic crystal fibers. In OFC Proceedings (Vol. paper OME4). IEEE.

\section{General rights}

Copyright and moral rights for the publications made accessible in the public portal are retained by the authors and/or other copyright owners and it is a condition of accessing publications that users recognise and abide by the legal requirements associated with these rights.

- Users may download and print one copy of any publication from the public portal for the purpose of private study or research.

- You may not further distribute the material or use it for any profit-making activity or commercial gain

- You may freely distribute the URL identifying the publication in the public portal 


\title{
Optical tuning of photonic bandgaps in dye-doped nematic liquid crystal photonic crystal fibers
}

\author{
Thomas Tanggaard Alkeskjold and Anders Bjarklev \\ COM Research Center, Technical University of Denmark, DK-2800 Lyngby, Demmark, ul@com.dtu.dk. \\ David Sparre Hermann \\ Photonics Laboratory, Department of Microtechnology and Nanoscience MC2, Chalmers University of Technology, 41296 Gothenburg, Sweden. \\ Jes Broeng \\ Coystal Fibre A/S, BIokken 84, DK-3460 Birkered, Denmark.
}

\begin{abstract}
An all-optical modulator is demonstrated, which utilizes a pulsed $532 \mathrm{~nm}$ laser to modulate the spectral position of the bandgaps in a photonic crystal fiber infiltrated with a dye-doped nematic liquid crystal. (c) 2005 Optical Society of America

OCIS codes: (060.2310) Fiber Optics; (230.3990) Microstructure Devices; (230.3720) Liquid Crystal Devices
\end{abstract}

\section{Introduction}

Photonic crystal fibers (PCFs) have attracted significant attention during the last years and much research has been devoted to develop fiber designs for various applications, hereunder tunable fiber devices. This type of devices were first demonstrated by Eggleton et al. [1], where a high-index liquid plug was dynamically positioned in a tapered 'grapefiuit' fiber, thereby creating a fiber device with tunable transmission properties. Later, Bise et al. [2] demonstrated a tunable photonic bandgap fiber, where the air holes of a solid-core PCF was filled with a high-index oil. Recently, thermally tunable fiber devices based on Liquid Crystals (LCs) was demonstrated [3], where tunable and switchable bandgaps was demonstrated. Here we present an all-optical tunable Liquid Crystal Photonic BandGap (LCPBG) fiber device, which utilizes a pulsed 532nm laser to modulate the spectral position of the bandgaps in a PCF infiltrated with a dye-doped nematic liquid crystal. A modulation frequency of $2 \mathrm{kHz}$ is demonstrated for a pump power of only $2-3 \mathrm{~mW}$.

\section{Optically tuned LCPBG fibers.}

In this experiment we have used a so-called 'large-mode area' PCF, which is an all-silica PCF, which has a silica core surrounded by 7 periods of air holes arranged in a triangular lattice. The hole diameter, inter hole distances and core size is $3 \mu \mathrm{m}, 7 \mu \mathrm{m}$ and $10 \mu \mathrm{m}$, respectively. The fiber is endlessly single mode and guides light by the principle of Total-Internal-Reflection (TIR). When infiltrating the air holes of the PCF with a LC, which has a higher refractive index than that of silica, the infiltrated section cannot guide by TIR, but can support a finite number of guided wavelength bands due to anti-resonant reflection from the LC-filled holes [3]. The spectral position of these bandgaps is determined by the alignment of the LC, the structural parameters of the PCF and refractive indices of the LC. Since the refractive indices of the LC is considerably higher than that of silica, the LC infiltrated holes can be approximated as isolated waveguides and the spectral position of the bandgaps becomes insensitive to the inter hole distance and is dominated by the features of the imner ring of holes surrounding the core [4,5]. The transmission losses within the bandgaps are reduced when increasing the number of periods surrounding the core, and necessary for achieving good transmission. Having fixed the PCF parameters in the manufacturing process, the bandgaps can be tuned by changing the orientation or/and modifying the refractive indices of the LC. The refractive indices of a LC can be easily tuned using the high thermo-optic effect of most nematic LCs. The temperature dependent refractive indices of the nematic LC used in this experiments (E7, Merck, Darmstadt, Germany) is shown in figure 1. The refractive indices are shown for $\lambda=450 \mathrm{~nm}$ and for $\lambda=656 \mathrm{~nm}$ and the experimental data is fitted using a four parameter model developed by Jun $\mathrm{Li}$ et al. [6] (Jun $\mathrm{Li}$ is acknowledged for providing experimental data on $\mathrm{E} 7$ ).

The alignment of the LC mesogens in the PCF was determined by polarized microscopy of a single fused-silica capillary tube infiltrated with the LC. The LC was homogeneously aligned with the LC director pointing in the direction of the fiber axis. Due to this alignment, the spectral position of the bandgaps is dominated by the ordinary refractive index of $E 7\left(n_{0}\right)$, which is the refractive index seen in the transverse plane of the PCF, but a contribution from the extraordinary index $\left(\mathrm{n}_{\mathrm{e}}\right)$ should also be expected due to a non-zero z-component of the cladding mode field. 


\section{OME4}
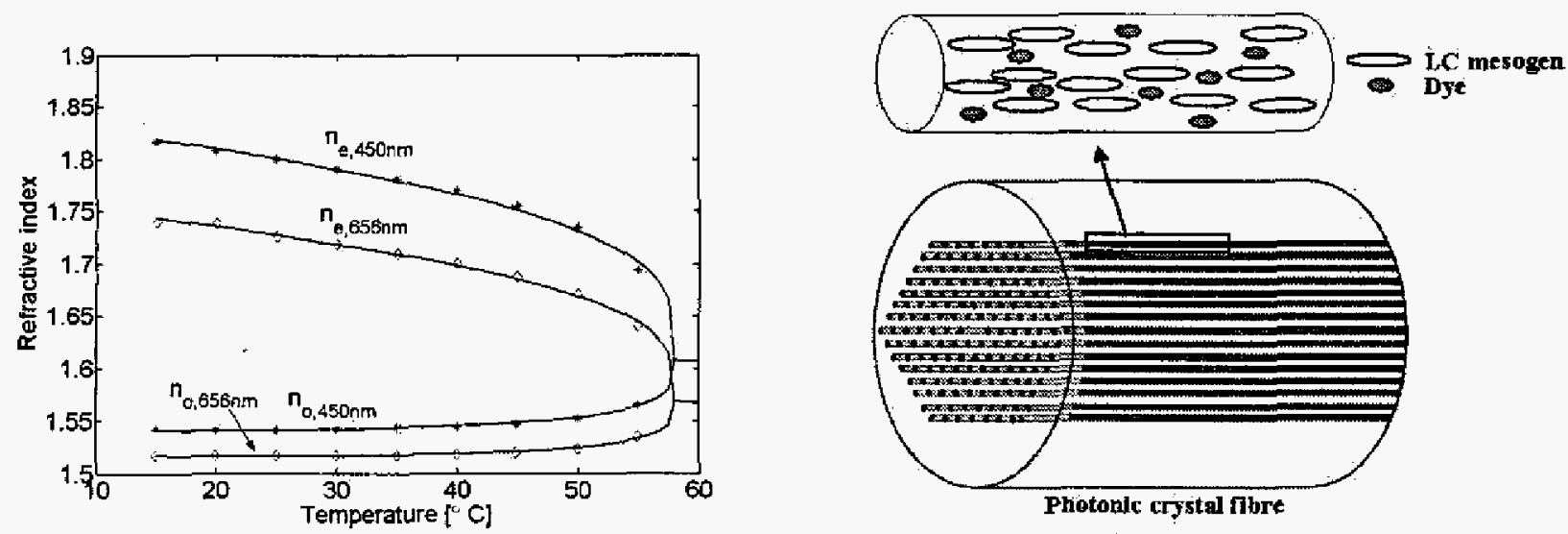

Figure 1 Left: Temperature dependence of the refractive indices of LC E7. Experinental data points is provided by Jun $1 \dot{j}$ and the temperature dependence is fitted using a four-parameter nodel by Jun Li [4]. Right: The air holes of a PCF is infiltrated with a dye doped LC ( $E 7$, Merck), and by using polarized microscopy on a single capillary tube, a homogeneously alignment of the LC were observed.

The tuning sensitivity is therefore linked to the temperature gradient of $n_{0}$ and from figure 1 (left) it is seen that the temperature gradient of $n_{0}$ is close to zero around room temperature but becomes positive, when the temperature is increased to above approx. $35^{\circ} \mathrm{C}$. The air holes of a $40 \mathrm{~m}$ long PCF were infiltrated with E7 for $10 \mathrm{~mm}$ of the fiber Iength and the photonic bandgaps were measured using an unpolarized halogent-tungsten source. The bandgaps from $450 \mathrm{~nm}$ to $1750 \mathrm{~nm}$ at $40^{\circ} \mathrm{C}$ is shown on the top left figure of figure 2 , and the temperature dependence of the spectral position of the two bandgaps around $540 \mathrm{~nm}$ and $1400 \mathrm{~nm}$ is shown on figure 2 (right).

In order to decrease the thermal response time, compared to heating/cooling the entire fiber, a $532 \mathrm{~nm}$ pulsed laser is used to locally heat the LC positioned in the inner ring of holes surrounding the core. The heating efficiency is increased when there is a high overlap between the core mode and the inner ring of cladding holes. This is achieved by heating the entire fiber using a hotplate, such that the $532 \mathrm{~nm}$ source is positioned on the edge or just outside a bandgap. On the bandgap edges, the effective area of the core mode increases substantially [7], thereby leading to a higher overlap between the core mode and the LC in the inner ring of holes. As seen from figure 2 (right) the 532nm laser will be positioned on the bandgap edge or outside a bandgap when the LC temperature is between $44^{\circ} \mathrm{C}$ and $52^{\circ} \mathrm{C}$. Further, increasing the absorption of the $\mathrm{LC}$ around $532 \mathrm{~nm}$ can increase the temperature sensitivity. This is achieved by doping the LC with a dye. We therefore doped E7 with an azobenzene dye called Disperse Red 1 $(0.4 \mathrm{wt} \%)$. The dye DRI has an absorptions peak around $502 \mathrm{~nm}$ and the dye doping does not affect the alignment of the LC. Figure 2 (left) shows the bandgaps of the LCPBG fiber before and after dye doping. The figure clearly shows that the presence of the dye, decreases the transmission in the bandgaps located around the dye absorption peak, but does not alter the position or the transmission level of the remaining bandgaps.
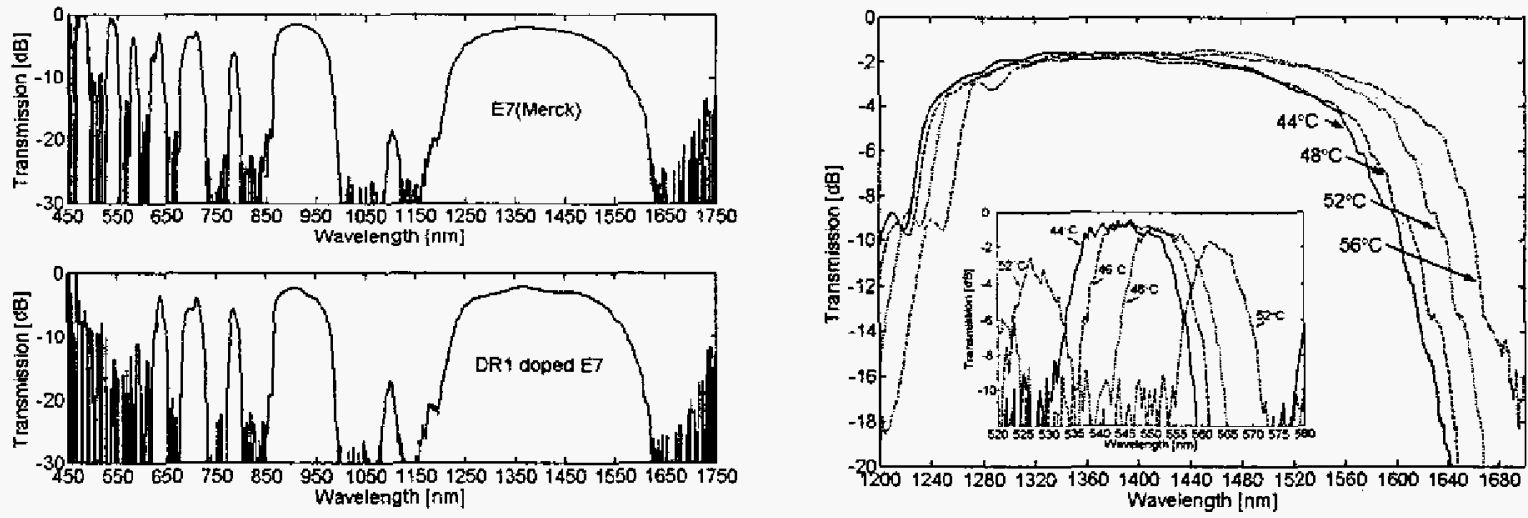

Figure 2 Left: Transmission spectrum of a nenatic LCPBG fiber infiltrated for $10 \mathrm{~mm}$ with E7. Spectrum is without and with dye doping with DR 1 . Right: Temperature dependence of the bandgaps around $540 \mathrm{~nm}$ and $1400 \mathrm{~mm}$ in a LCPBG fiber without dye doping. 

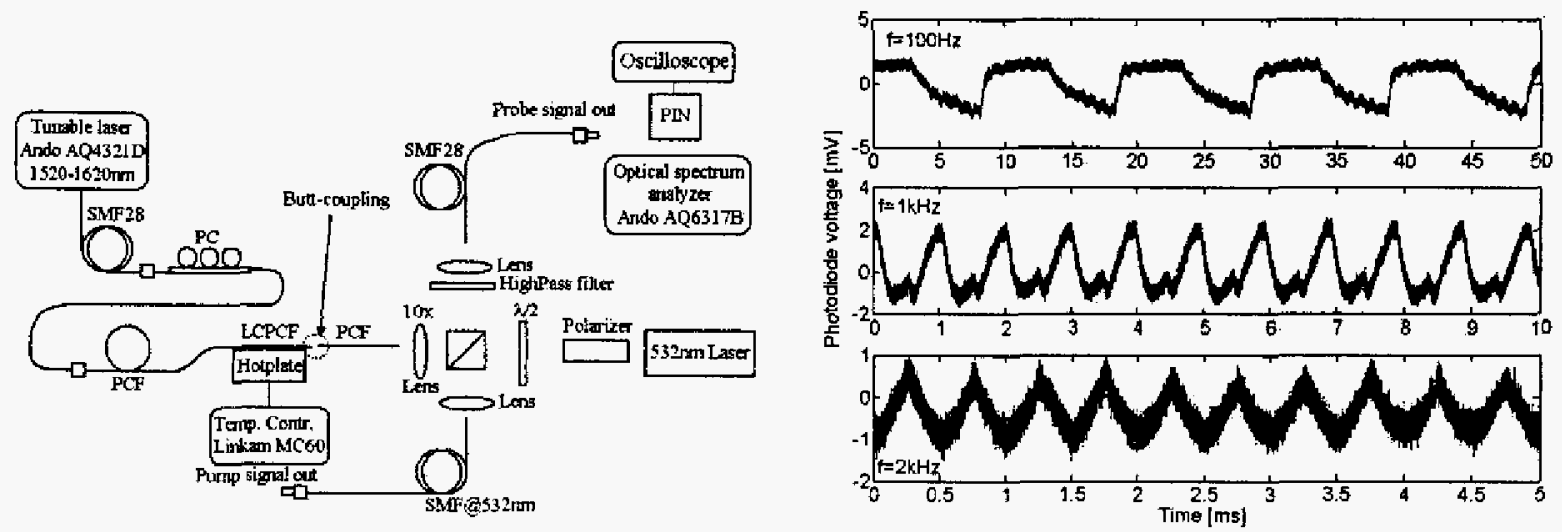

Figure 3 Ieft: Experimental setup for measuring the time response of the probe laser. Right: Oscilloscope traces of the $1620 \mathrm{~nm}$ probe laser when the $532 \mathrm{~nm}$ pump laser is modulated with $100 \mathrm{~Hz}, 1 \mathrm{kHz}$ and $2 \mathrm{kHz}$.

In order to investigate the time response of the LCPBG fiber device, a low-power CW probe laser was coupled into the fiber together with the pulsed pump laser. Both the polarization of the pump and the probe could be individually adjusted using the setup shown on figure 3 (left). The probe laser was chosen to be located on the long-wavelength side of the bandgap centered around $1400 \mathrm{~nm}$ (as shown on figure 2, right). In the center of the bandgap, no polarization sensitivity was observed on the transmission level, while this was not the case on the edge of the bandgap. Using a polarized tunable laser, we measured the polarization sensitive bandgap edge from 1520-1620nm and observed a small shift (approx. 10nm) of the bandgap edge depending on the polarization of the laser. This shift is attributed to very small variations (few tens of nanometers) in the hole diameter and shape of the inner ring of holes, thereby leading to small birefringence. When operating the probe laser on this edge, we therefore observed sensitivity to both the polarization of the purnp and the probe laser, but we will not discuss this further in this paper. The temperature of the LCPBG fiber was raised to $45^{\circ} \mathrm{C}$ and the probe laser was monitored using a PIN photo detector and a storage oscilloscope. The pulsed pump laser (square-wave modulated, $50 \%$ duty cycle) was turned on and the polarization of both the pump and probe laser was adjusted until a modulation of the probe laser was observed on the oscilloscope. Figure 3 (right) shows the observed waveforms of the $1620 \mathrm{~nm}$ probe laser when the $532 \mathrm{~nm}$ pump laser were modulared with $100 \mathrm{~Hz}, 1 \mathrm{kHz}$ and $2 \mathrm{kHz}$ square wave signal. The power of the pump laser was monitored and we estimate that the power of the pump coupled into the LCPBG device, in the junction of the butt-coupling, was around $2-3 \mathrm{~mW}$ peak power. Increasing the input power did not yield any improvement, which is attributed to a too large increase in temperature, whereby a bandgap shift up in wavelength and assist in confining the pump to the core region, and hence decrease the overlap with the LC (see inset on figure 2, right). We believe that these optically tuned LCPBG fiber devices can find use as distributed devices i.e. with signal processing elements, such as tunable bragg gratings, distributed in a transmission fiber and with the data signal (the probe) and the control signal (the pump) carried in the same fiber.

\section{Conclusion}

In this paper we have, to our knowledge, presented the first optical tunable PBG fiber device. We have demonstrated how a $532 \mathrm{~nm}$ pulsed laser can modulate a $1620 \mathrm{~nm} \mathrm{CW}$ laser by locally modifying the optical properties of LC inclusions in a LC infiltrated PCF. This is achieved using a low pump power of only $2-3 \mathrm{~mW}$, and we have demonstrated a modulation frequency of up to $2 \mathrm{kHz}$.

\section{References}

[1] B.J. Eggleton, C. Kerbage, P.S. Westbrook, R.Windeler \& A. Hale, "Microstructured optical fiber devices", Opt. Express 9, 698-713(2001). [2] R. T. Bise, R. S. Windeler, K. S. Kranz, C. Kerbage, B. J. Eggleton, and D. J. Trevor, "Tunable photonic band gap fiber," Optical Fiber Conmunication Conference Technical Digest, pp. 466-468 (2002).

[3] T. T. Larsen, A. Bjarklev, D. S. Hermann, and J. Broeng, "Optical devices based on liquid crystal photonic bandgap fibres," Opt. Express 11, $2589-2596(2003)$.

[4] A. K. Abeeluck, N.M. Litchenitser, C. Headley and B. J. Eggelton, "Analysis of spectral characteristics of photonic bandgap waveguides", Opt. Express 10, 1320-1333 (2002)

[5] N. M. Litchinitser. S. C. Dunn, P. E. Steinvurzel, B. J. Eggleton, T. P. White, R. C. McPhedran, and C. M. de Sterke, "Application of an ARROW model for designing tunable photonic devices," Opt. Express 12, 1540-1550 (2004).

[6] J. Li, S. Gauza and S. Wu, "Temperature effect on liquid crystal refractive indices", Jour. of Appl. Phys. Vol. 96, No. 1 (2004).

[7] J. Rishede, J. Lxgsgaard, J. Broeng and A. Bjarklev, "All-silica photonic bandgap fibre with zero dispersion and a large mode area at $730 \mathrm{~nm}$ ", J. Opt. A: Pure Appl. Opt. 6 (2004) 667-670 